\title{
ON THE MODULATIONAL STABILITY OF GROSS-PITTAEVSKII TYPE EQUATIONS IN 1+1 DIMENSIONS
}

\author{
Z. RAPTI \\ Department of Mathematics and Statistics, University of Massachusetts, \\ Amherst MA 01003-4515, USA \\ E-mail: rapti@math.umass.edu \\ P. G. KEVREKIDIS \\ Department of Mathematics and Statistics, University of Massachusetts, \\ Amherst MA 01003-4515, USA \\ E-mail:kevrekid@math.umass.edu \\ V. V. KONOTOP \\ Centro de Física de Matéria Condensada, Universidade de Lisboa, Av. Prof. \\ Gama Pinto, 2, Lisboa 1649-003, Portugal \\ E-mail: konotop@cii.fc.ul.pt
}

\begin{abstract}
The modulational stability of the nonlinear Schrödinger (NLS) equation is examined in the cases with linear and quadratic external potential. This study is motivated by recent experimental studies in the context of matter waves in BoseEinstein condensates (BECs). The linear case can be examined by means of the Tappert transformation and can be mapped to the NLS in the appropriate (constant acceleration) frame. The quadratic case can be examined by using a lens-type transformation that converts it into a regular NLS with an additional linear growth term.
\end{abstract}

\section{Introduction}

Intensive studies of Bose-Einstein condensates (BECs) ${ }^{1}$ have drawn much attention to nonlinear excitations in them. Recent experiments have revealed the existence of bright ${ }^{2}$ and dark ${ }^{3,4,5}$ solitons in BECs, as well as topological structures, such as vortices ${ }^{6}$ and vortex lattices ${ }^{7}$. An interesting question concerns how such solitary wave structures may arise in this novel context of matter waves in BECs. It is well-known that the dynamics of the wavefunction in BEC is described (at the mean field level, 
which is an increasingly accurate description, the closer the system is to the zero temperature limit) by the Gross-Pittaevskii (GP) equation which is a variant of well-known nonlinear Schrödinger (NLS) equation ${ }^{8}$. In the NLS context, perhaps the most standard mechanism through which solitons and solitary wave structures appear is through the activation of the modulational instability (MI) of plane waves.

The MI is a general feature of discrete as well as continuum nonlinear wave equations. Its demonstrations span a diverse set of disciplines ranging from fluid dynamics ${ }^{9}$ (where it is usually referred to as the Benjamin-Feir instability) and nonlinear optics ${ }^{10}$ to plasma physics ${ }^{11}$. One of the early contexts in which its significance was appreciated was the linear stability analysis of deep water waves.

The MI has been examined recently in the context of optical lattices in BECs both in one-dimensional and quasi-one dimensional systems, as well as in multiple dimensions. In the former case, it has been predicted theoretically ${ }^{12,13}$ and verified experimentally ${ }^{14,15}$ to lead to destabilization of plane waves, and in turn to delocalization in momentum space (equivalent to localization in position space, and the formation of solitary wave structures).

In the present contribution, we discuss the MI conditions for the continuous NLS equation (or equivalently for the GP equation) in $(1+1)$ dimensions ( 1 spatial and 1 temporal)

$$
i u_{t}+u_{x x}+s|u|^{2} u+V(x) u=0 .
$$

$u$ in this equation describes the slow envelope complex field dynamics (modulating the fast oscillatory dynamics). The subscripts denote partial derivatives with respect to the index, $s \in\{1,-1\}$ illustrates the focusing $(+1)$ or defocusing $(-1)$ nature of the nonlinearity, while $V(x)$ is the external potential.

In section 2.1, we review briefly the results in the absence of the potential (e.g., for $V(x) \equiv 0$ ). In section 2.2 , we examine the case of a linear potential:

$$
V(x)=-\alpha x,
$$

which is relevant in experimental situations with gravitational ${ }^{16}$ (and potentially also electrostatic) fields. In section 2.3 , we examine the quadratic potential of the general form:

$$
V(x)=-k(t) x^{2}
$$

which is relevant to contexts in which the (magnetic) trap is strongly confined in the 2 directions, while it is much shallower in the third one ${ }^{1}$. 
The prefactor $k(t)$ is typically fixed in current experiments, but adiabatic changes in the strength (and in fact even the location of the center) of the trap are experimentally feasible, hence we examine the more general time-dependent case. Finally, in section 3 we summarize our findings and conclude.

\section{Analytical Results}

\subsection{No potential}

We start by recalling the results for the modulational stability of the NLS (1) without an external potential, i.e. for $V(x) \equiv 0$ : To this end we look for perturbed plane wave solutions of the form

$$
u(x, t)=(\phi+\epsilon b) \exp [i((q x-\omega t)+\epsilon \psi(x, t))]
$$

and analyze the $O(\epsilon)$ terms as

$$
b(x, t)=b_{0} \exp (i \beta(x, t)), \quad \psi(x, t)=\psi_{0} \exp (i \beta(x, t)) .
$$

Using $\beta(x, t)=Q x-\Omega t$, the dispersion relation connecting the wavenumber $Q$ and frequency $\Omega$ of the perturbation (see e.g. ${ }^{8}$ )

$$
(-\Omega+2 q Q)^{2}=Q^{2}\left(Q^{2}-2 s \phi^{2}\right)
$$

is obtained.

This implies that the instability region for the NLS in the absence of an external potential, appears for perturbation wavenumbers $Q^{2}<2 s \phi^{2}$, and in particular only for focusing nonlinearities (to which we will restrict our study from this point onwards).

\subsection{Linear Potential}

The case of a linear potential is relevant to any context of a costant external field (gravitational ${ }^{16}$ and electric ones being among the prominent such examples). In this setting, the NLS is well-known to maintain its inte-

grable character ${ }^{17}$. Hence, in some sense, we expect that the modulational results/conditions will not be modified in this case.

The simplest way to illustrate that is by means of the "Tappert transformation" $17\left(\xi=x+\alpha t^{2}\right)$

$$
u(x, t)=v(\xi, t) \exp \left(-i \alpha x t-\frac{1}{3} i \alpha^{2} t^{3}\right)
$$


(notice however the difference with the expression proposed in ${ }^{17}$ ) which brings the Eq. (1) back into the form of the regular NLS equation, without the external potential, in which case the condition of Eq. (6) applies.

Hence, the growth terms will now be $\sim \exp (i(Q \xi-\Omega t))$ with $\Omega=\Omega_{r}+i \nu$ (when the instability condition is satisfied; $\Omega_{r}=2 q Q$, cf. Eq. (6)), and hence the instability will be developing according to the spatiotemporal form:

$$
u \sim \exp \left(i Q\left(x+\alpha t^{2}\right)-i \Omega_{r} t+\nu t-i \alpha x t-\frac{1}{3} i \alpha^{2} t^{3}\right) .
$$

\subsection{Quadratic Potential}

The quadratic potential of Eq. (3) is clearly the most physically relevant example of an external potential in the BEC case, given the harmonic confinement of the atoms by the experimentally used magnetic traps ${ }^{1}$. In particular, to examine the MI related properties in this case, we will use a lens-type transformation ${ }^{8}$ of the form:

$$
u(x, t)=\ell^{-1} \exp \left(\text { if }(t) x^{2}\right) v(\zeta, \tau)
$$

where $f(t)$ is a real function of time, $\zeta=x / \ell(t)$ and $\tau=\tau(t)$. To preserve the scaling we choose ${ }^{8,18}$

$$
\tau_{t}=1 / \ell^{2}
$$

To satisfy the resulting equations, we then demand that:

$$
\begin{gathered}
-f_{t}=4 f^{2}+k(t) \\
-\ell_{\tau} / \ell+4 f \ell^{2}=0 .
\end{gathered}
$$

Taking into account (10) the last equation can be solved:

$$
\ell(t)=\ell(0) \exp \left(4 \int_{0}^{t} f(s) d s\right)
$$

what reduces the problem of finding time dependence of the parameters to solution of Eq. (11).

Upon the above conditions, the equation for $v(\zeta, \tau)$ becomes

$$
i v_{\tau}+v_{\zeta \zeta}+|v|^{2} v-2 i \lambda v=0
$$

where

$$
f \ell^{2}=\lambda
$$


and generically $\lambda$ is real and depends on time. Thus we retrieve NLS with an additional term, which represents either growth (if $\lambda>0$ ) or dissipation (if $\lambda<0$ ).

Eq. (14) allows an explicit solution when $v \equiv v(\tau)$ (i.e., a spatially homogeneous solution). The latter is of the form

$$
\begin{aligned}
& v=\frac{A_{0}}{\ell(t)} \exp \left(i f(t) x^{2}+i q \frac{x}{\ell(t)}-i q^{2} \tau(t)\right) \times \\
& \exp \left(\Lambda(\tau)+i \theta_{0}+i A_{0}^{2} \int_{0}^{\tau} \exp (2 \Lambda(s)) d s\right),
\end{aligned}
$$

where $\Lambda(\tau)=4 \int_{0}^{\tau} \lambda(s) d s$, and $A_{0}$ and $\theta_{0}$ are arbitrary real constants.

A particularly interesting case is that of $\lambda$ constant. Then from the system of equations (10)-(12) and (14) it follows that $k$ must have a specific form. $f, \ell$ and $\tau$ can then be determined accordingly. In fact, the system (10)-(12) and (14) with $\lambda$ constant has as its solution

$$
\begin{aligned}
k(t) & =\left(t+t^{*}\right)^{-2} / 16 \\
f(t) & =\left(t+t^{*}\right)^{-1} / 8 \\
\ell(t) & =2 \sqrt{2 \lambda} \sqrt{t+t^{*}} \\
\tau(t) & =\ln \left(\frac{t+t^{*}}{t^{*}}\right) / 8 \lambda .
\end{aligned}
$$

Notice once again, per the assumption of an imaginary phase in the exponential of Eq. (9), that our considerations are valid only for $\lambda \in R$. $t^{\star}$ in the above equations is an arbitrary constant that essentially determines the "width" of the trap at time $t=0$ according to Eq. (17).

In this case the modulational condition remains unchanged, but now $\omega$ satisfies the dispersion relation $\omega=q^{2}-\phi^{2}+2 \imath \lambda$, so the growth (if $\lambda>0$ ) or dissipation (if $\lambda<0$ ) is inherent in equation (4). Moreover, all the terms are modulated by the constant growth (or decay) rate $\exp (2 \lambda \tau)$, and the instability (when present) will be developing according to the form $v \sim \exp \left(i\left(Q \zeta-\Omega_{r} \tau\right)+(\nu+2 \lambda) \tau\right)$ with $\Omega=\Omega_{r}+i \nu . \Omega_{r}=2 q Q$.

If $k=k(t)$ is not given by Eq. (17), then $\lambda$ must be time dependent (e.g., $\lambda \equiv \lambda(t))$. Here one cannot directly perform the MI analysis. However, still in this case, we have converted the explicit spatial dependence into an explicit temporal dependence. An important conclusion that stems from this transformation is that the harmonic potential, viewed in the appropriate frame (of Eq. (9)) can be considered as a form of growth (or dissipation, depending on the sign of $\lambda$ for $\lambda \in R$ ) term. 


\section{Conclusions}

In this brief communication, we have examined the problem of modulational instabilities of plane waves in the context of Gross-Pittaevskii equations with an external (linear or quadratic) potential. Both of these cases are directly relevant to current experimental realizations of Bose Einstein condensates.

It was found that the linear (also integrable) case can be reverted to the original NLS setting (wherein the MI conditions are well-known) by an appropriate (Tappert) transformation. This transformation was used to develop the form of the growth of unstable wavenumbers. Notice, however, that these are the wavenumbers of the expansion in a novel spatial coordinate which is essentially the spatial variable in a frame with constant acceleration. Hence, in this case, in the frame moving with a constant acceleration (induced by the constant force), the problem resumes its original NLS format and the MI conditions and resulting growth can be obtained in that frame and then restored in the original frame.

For the case of the quadratic potential, a lens transformation was used to cast the problem in a rescaled space and time frame (in a way very reminiscent of the scaling in problems related to focusing ${ }^{8,18}$ ). In this rescaled frame, the external potential can be viewed as a form of external growth. For specific forms of temporal dependence of the prefactor of the harmonic term (e.g., for appropriate, non-autonomous quadratic potentials), the resulting prefactor is constant. In such a context once again the MI analysis can be carried through completely, producing similar conditions, but now in the new dynamically rescaled frame/variables (which can be appropriately re-cast in the original variables).

It would be interesting to examine if similar considerations can be generalized to the case of multiple dimensions. Furthermore, in the current scheme of things it seems that the cases of different forms of the potentials need to be treated in different ways (which can be understood in terms of the different physical effects that they represent). Nonethless, it would be very useful, if a general formulation could be developed that could be applied independently of the form of $V(x)$, having as special case limits, the potential forms presented herein.

Finally, it would be worth exploring whether the explicitly demonstrated as modulationally unstable settings presented herein can be used as a means (i.e., initial condition) for directly producing solitary (matter) wave structures in BECs in an alternative fashion to the ones currently used in BEC 
experiments.

Prof. D.J. Frantzeskakis is gratefully acknowledged for a critical reading of the manuscript and his suggestions. PGK gratefully acknowledges support from a University of Massachusetts Faculty Research Grant, from the Clay Foundation through a Special Project Prize Fellowship and from the NSF through DMS-0204585. VVK gratefully acknowledges support from the European grant COSYC n.o HPRN-CT-2000-00158.

\section{References}

1. F. Dalfovo, S. Giorgini, L. P. Pitaevskii, and S. Stringari, Rev. Mod. Phys. 71, 463(1999).

2. K.E. Strecker et al., Nature 417, 150 (2002); L. Khaykovich et al., Science 296, 1290 (2002).

3. S. Burger et al., Phys. Rev. Lett. 83, 5198 (1999).

4. J. Denschlag et al., Science 287, 97 (2000).

5. B.P. Anderson et al., Phys. Rev. Lett. 86, 2926 (2001).

6. M.R. Matthews et al., Phys. Rev. Lett. 83, 2498 (1999); K.W. Madison et al. Phys. Rev. Lett. 84, 806 (2000); S. Inouye et al., Phys. Rev. Lett.87, 080402 (2001).

7. J.R. Abo-Shaeer et al, Science 292, 476 (2001); J.R. Abo-Shaeer, C. Raman and W. Ketterle, Phys. Rev. Lett. 88, 070409 (2002); P. Engels et al, Phys. Rev. Lett. 89, 100403 (2002).

8. C. Sulem and P.L. Sulem, The Nonlinear Schrödinger Equation, SpringerVerlag (New York, 1999).

9. T.B. Benjamin and J.E. Feir, J. Fluid. Mech. 27, 417 (1967).

10. L.A. Ostrovskii, Sov. Phys. JETP 24, 797 (1969).

11. T. Taniuti and H. Washimi, Phys. Rev. Lett. 21, 209 (1968); A. Hasegawa, Phys. Rev. Lett. 24, 1165 (1970).

12. V.V. Konotop and M. Salerno, Phys. Rev. A 65, 021602(R) (2002).

13. A. Smerzi, A. Trombettoni, P.G. Kevrekidis, and A.R. Bishop, condmat/0207172 (Phys. Rev. Lett., in press).

14. F.S. Cataliotti et al., cond-mat/0207139.

15. M. Kasevich and A. Tuchman (private communication).

16. B. P. Anderson and M. A. Kasevich, Science, 282, 1686 (1998).

17. H.-H. Chen and C.-S. Liu, Phys. Rev. Lett., 37, 693 (1976).

18. see e.g., S.I. Siettos, I.G. Kevrekidis and P.G. Kevrekidis, nlin.PS/0204030 and references therein. 